\title{
Reusable Nanocatalyst: Zirconia and Sulfated Zirconia
}

\author{
Mitesh B. Gondaliya ${ }^{*}$, Tushar Maheta, Mayank J. Mamtora, Manish K. Shah \\ Department of Chemistry, Saurashtra University, Rajkot, Gujarat, India \\ *E-mail address: miteshgondaliya@scientist.com
}

\begin{abstract}
1,5-benzodiazepines have been synthesized by Nano Zirconia and Nano Sulfated Zirconia in solvent free conditions. Synthesis was carried out by conventional as well as microwave method. Benzodiazepines were synthesized by reacting o-phenylenediamine and few ketones in the presence of catalytic amount of Nanoparticles. Particles used were sized in between 53-100 nm. Nanoparticles were characterized by FT-IR, DLS and powder XRD analysis.
\end{abstract}

Keywords: Nanocatalyst; Nanoparticles; Sulfated Zirconia; Synthesis; Benzodiazepines; Solid catalyst; Solvent free; Green Chemistry

\section{INTRODUCTION}

Benzodiazepines is very well known drug which gives various pharmacological importance, for example anxiolytic, anticonvulsant, hypnotic and sedative agent [1,2]. So benzodiazepines were of great interest in industries. There were many methods known, which were considered for up-gradation in urgencies. Many catalyst reported in literatures with significant boundaries were Silica supported 12-tungstophosphoric acid [3], boron trifluoride ether complex [4], Ytterbium triflate [5], Magnesium oxide/phosphorus oxychloride [6]. All methods in literature has its distinct use and also has some limitations like green aproach, reusability, etc. The most prominent method is to use solid superacid catalyst, which can be further be recovered and reused several times. While, if we use nanomaterial, which have greater surface area compared to macro particles, may give faster reaction. So, taking in mind, we have used Nano Zirconia and Nano sulfated Zirconia for the synthesis of benzodiazepines.

\section{EXPERIMENTAL}

Thin-layer chromatography was accomplished on $0.2 \mathrm{~mm}$ precoated plates of silica gel G60 F254 (Merck). IR spectra were recorded on a FTIR-8400 spectrophotometer using DRS prob. ${ }^{1} \mathrm{H}(400 \mathrm{MHz})$ and ${ }^{13} \mathrm{C}(100 \mathrm{MHz}) \mathrm{NMR}$ spectra were recorded on a Bruker ADVANCE 
II spectrometer in DMSO-d6. Chemical shifts are expressed in $\delta$ ppm downfield from TMS as an internal standard. Solvents were evaporated with a BUCHI rotary evaporator. Melting points were measured in open capillaries and are uncorrected. X-ray diffraction (XRD) measurements were performed on the XPERT-MPD by an X-ray diffractometer using $\mathrm{Cu} \mathrm{K} \alpha$ radiation source $(\lambda=1.5418)$ at power $200 \mathrm{~kW}$. XRD Crystallite Size calculation was done using Scherrer equation from powder XRD data. Particle size mesearments were carried out on DLS Nanotrac of Microtrac Europe GmbH. Domastic microwave was used at $400 \mathrm{w}$. power Preparation of Zirconia and Sulfated Zirconia were adapted ${ }^{[7,8]}$ and modified.

\section{1. Preparation of zirconia catalyst}

Hydrated Zirconium oxychloride was dissolve in water then added ammonium hydroxide till solution become basic. The precipitate obtained was filtered. Zirconiumhydroxide gel was dried in oven then formed solid was crushed. The fine powder was calcinated at $600{ }^{\circ} \mathrm{C}$ for 4 hour. The particle obtain were stored in cold and anhydrous environment.

\section{2. Preparation of Sulfated-Zirconia}

Previously prepared Zirconium hydroxide was acidified with $0.1 \mathrm{M}$ sulfuric acid then stirred for 24 hour then centrifuged and dried in oven then formed solid was crushed. The fine powder was calcinated at $600{ }^{\circ} \mathrm{C}$ for 4 hour. The particle obtain were charectorised by XRD, DLS and FT-IR.

\section{3. General procedure for synthesis of Benzodiazepines}

A mixture of o-phenylenediamine $(1 \mathrm{mmol})$, different ketone $(2.5 \mathrm{mmol})$ and a catalytic amount of nanomaterial $(0.02 \mathrm{mg})$ in round bottom flask were stirred at ambient condition. Reaction was monitered by TLC in intervals. After complition of reaction the nanoparticles were recovered by adding dichloromethane $(10 \mathrm{ml})$ and then centrifuged in high RPM untill nanoparticles were setteled in bottom. Washed nanoparticles 2-3 times with dichloromethane and nanoparticles were reused in reaction wet/dried. Similarly reactions were carried out in microwave.

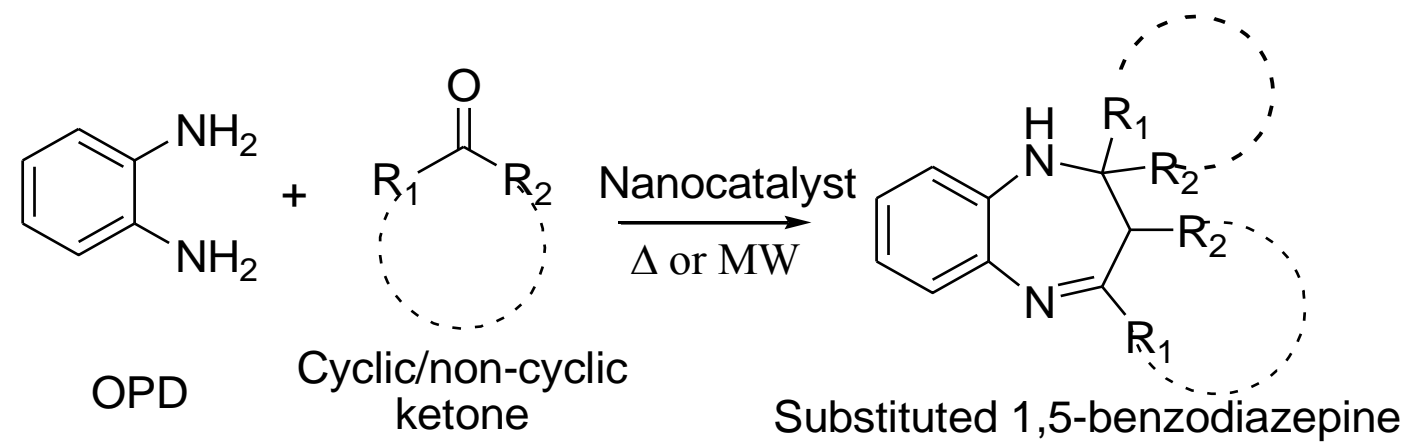

Scheme 1. General reaction for synthesis of 1,5-benzodiazepine derivatives.

2,2,4-trimethyl-2,3-dihydro-1H-benzo[b][1,4]diazepine (AZ-1): Yellow solid, mp 150-152 ${ }^{\circ} \mathrm{C}$; Mass $\mathrm{M}^{+}=188$; IR $(\mathrm{KBr}) v\left(\mathrm{~cm}^{-1}\right), 3330-\mathrm{NH}(\mathrm{s}, \mathrm{br}) ;{ }^{1} \mathrm{H}$ NMR $(\delta \mathrm{ppm}) 1.242(\mathrm{~s}, 6 \mathrm{H})$, 
$2.154(\mathrm{~s}, 2 \mathrm{H}), 2.230(\mathrm{~s}, 3 \mathrm{H}), 4.712(\mathrm{~s} \mathrm{br}, 1 \mathrm{H}), 6.825-6.965(\mathrm{~m}, 4 \mathrm{H}) ;{ }^{13} \mathrm{C} \mathrm{NMR}(\delta \mathrm{ppm}) 170.76$, $146.61,139.33,127.37,126.82$, 125.91, 121.08, 119.92, 79.29, 66.70, 45.15, 39.06, 29.38.

4,4'-(2-methyl-2,3-dihydro-1H-benzo[b][1,4]diazepine-2,4-diyl)diphenol (AZ-2): Yellow solid, mp 218-220 ${ }^{\circ} \mathrm{C}$; Mass $\mathrm{M}^{+}=344$; IR $(\mathrm{KBr}) v\left(\mathrm{~cm}^{-1}\right), 3330-\mathrm{NH}(\mathrm{s}, \mathrm{br}) ;{ }^{1} \mathrm{H}$ NMR $(\delta \mathrm{ppm})$ $1.630(\mathrm{~s}, 3 \mathrm{H}), 2.471(\mathrm{~d}, 1 \mathrm{H}), 4.712(\mathrm{brs}, 1 \mathrm{H}), 8.4(\mathrm{~s}, 1 \mathrm{H}), 8.87(\mathrm{~s}, 1 \mathrm{H}), 6.3-6.7(\mathrm{~m}, 4 \mathrm{H}), 6.8-7.2$ $(\mathrm{m}, 8 \mathrm{H}) ;{ }^{13} \mathrm{C} \mathrm{NMR}(\delta \mathrm{ppm}) 195.99,170.30,162.04,155.71,139.59,228.79,125.36,115.17$, 77.43, 59.76, 39.62, 30.18, 27.74, 20.61.

10-Spirocyclopentan-1,2,3,9,10,10a-hexahydrobenzo $[b]$-cyclopenta $[e][1,4]$ diazepine $(\mathrm{AZ}$ 3): Yellow solid, mp $138-140{ }^{\circ} \mathrm{C}$; Mass $\mathrm{M}^{+}=268$; IR $(\mathrm{KBr}) v\left(\mathrm{~cm}^{-1}\right), 3330-\mathrm{NH}(\mathrm{s}, \mathrm{br}) ;{ }^{1} \mathrm{H}$ NMR $(\delta \mathrm{ppm}) 1.2-1.8(\mathrm{~m}, 19 \mathrm{H}), 3.98(\mathrm{~s}$ br, $1 \mathrm{H}), 6.89-7.25(\mathrm{~m}, 4 \mathrm{H}) ;{ }^{13} \mathrm{C} \mathrm{NMR}(\delta \mathrm{ppm})$ $178.27,134.55,145.08,55.57,126.59$, 114.79, 121.80, 129.10, 43.54, 34.98, 37.07, 37.09, $22.56,27.48,22.51,22.53,25.1,22.68$.

2-methyl-2,4-diphenyl-2,3-dihydro-1H-benzo[b][1,4]diazepine (AZ-4): Yellow solid, mp 158-160 ${ }^{\circ} \mathrm{C}$; Mass $\mathrm{M}^{+}=312$; IR $(\mathrm{KBr}) v\left(\mathrm{~cm}^{-1}\right), 3330-\mathrm{NH}(\mathrm{s}, \mathrm{br}) ;{ }^{1} \mathrm{H}$ NMR $(\delta \mathrm{ppm}) 1.45(\mathrm{~s}$, $3 \mathrm{H}), 2.21(\mathrm{~s}, 2 \mathrm{H}), 4.02(\mathrm{~s} \mathrm{br}, 1 \mathrm{H}), 6.89-7.37(\mathrm{~m} 14 \mathrm{H}) ;{ }^{13} \mathrm{C}$ NMR $(\delta \mathrm{ppm}) 141.99,149.90$, $129.23,125.24,126.65,114.88,128.20,126.10,128.24,121.9,128.80,128.44,131.99$, $125.80,166.26,80.81,43.44,29.40$.

Table 1. Synthesis of 1,5-benzodiazepine derivatives.

Code Reactant Yield \%


AZ-4 Acetophenone

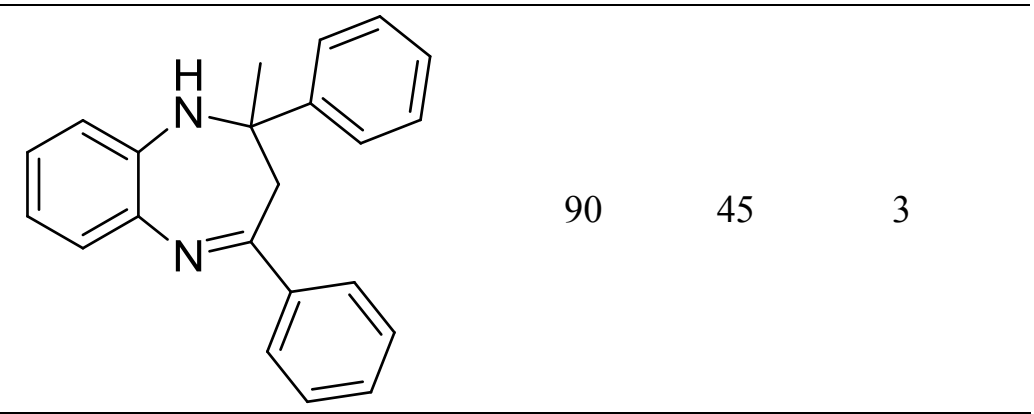

*yield \% isolated by crystallization in dichloromethane.

\section{RESULT AND DISCUSSION}

In this current work, we have synthesized Nano zirconium dioxide $\left(\mathrm{ZrO}_{2}\right)$ and sulfated zirconia. Both nanoparticles were studied for its catalytic activity to synthesis benzodiazepines derivatives. Nano particles were synthesized by solution gel method which is beneficiary in terms of bulk synthesis of Nano particles. The shape of Nano zirconium dioxide particles found from powder XRD were monoclinic and of Nano sulfated zirconia (SZ) particles were triclinic in shape. In particular, characterization we found that, in DLS, the size of nano zirconium dioxide was near about $100 \mathrm{~nm}$, and the similar size of Nano sulfated zirconia was also found near $100 \mathrm{~nm}$. From Powder XRD we found that the average bulk size of Nano zirconium dioxide was ranging between the 53-59 $\mathrm{nm}$ and geometrical structure was monoclinic. While, the average bulk size of Nano SZ was ranging between the 53-59 $\mathrm{nm}$ and geometrical structure was triclinic.

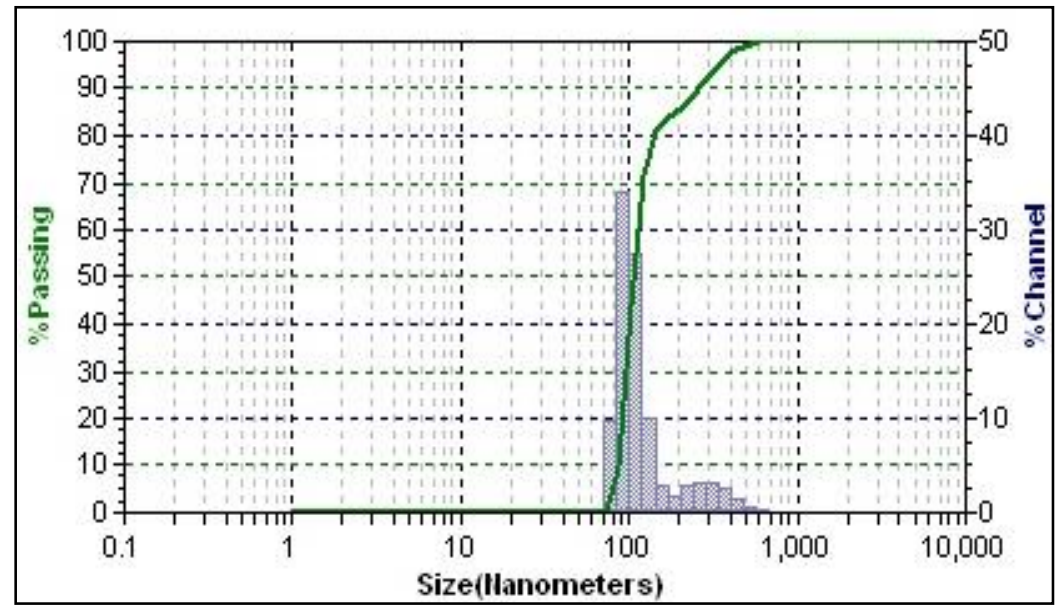

Summary
\begin{tabular}{|r|c|}
\hline Data & Value \\
\hline$M V(n m):$ & 366.0 \\
\hline MN(nm): & 137.7 \\
\hline MA(nm): & 278.9 \\
\hline CS: & 21.51 \\
\hline SD: & 39.90 \\
\hline PDI: & 0.931 \\
\hline Mz: & 363.9 \\
\hline$\sigma \imath:$ & 155.0 \\
\hline Ski:- 64.3926 \\
\hline Kg: & 1,060 \\
\hline
\end{tabular}

\begin{tabular}{|c|c|}
\multicolumn{2}{|c|}{$\%$ Tile } \\
\hline$\%$ Tile & Size $(\mathbf{n m})$ \\
\hline 95.00 & 341.0 \\
\hline 90.00 & 261.6 \\
\hline 80.00 & 139.4 \\
\hline 60.00 & 111.8 \\
\hline 50.00 & 105.6 \\
\hline 40.00 & 100.4 \\
\hline 30.00 & 95.80 \\
\hline 20.00 & 91.30 \\
\hline 10.00 & 86.10 \\
\hline 5.00 & 81.90 \\
\hline
\end{tabular}

Figure 1. DLS of $\mathrm{ZrO}_{2}$. 


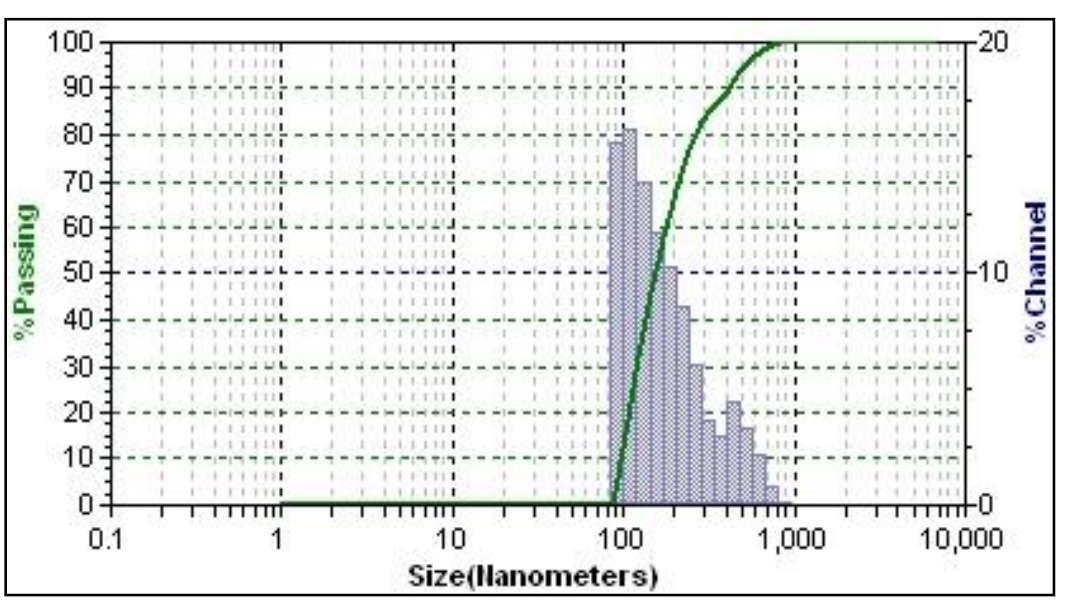

\begin{tabular}{|c|c|c|c|}
\hline \multicolumn{2}{|c|}{ Summary } & \multicolumn{2}{|c|}{ \%Tile } \\
\hline Data & Value & \%Tile & ize(nm) \\
\hline$M V(n m):$ & 513.0 & 95.00 & 519.0 \\
\hline$M N(n m):$ & 205.6 & 90.00 & 423.0 \\
\hline$M A(n m):$ & 415.0 & 80.00 & 266.0 \\
\hline cS: & 14.47 & 60.00 & 178.4 \\
\hline SD: & 102.0 & 50.00 & 153.1 \\
\hline PDI: & 0.759 & 40.00 & 133.9 \\
\hline Mz: & 507.1 & 30.00 & 119.0 \\
\hline$\sigma \mathrm{t}:$ & 196.3 & 20.00 & 106.8 \\
\hline Ski:- & 120.23666 & 10.00 & 97.00 \\
\hline $\mathrm{Kg}$ : & 1,042 & 5.00 & 92.20 \\
\hline
\end{tabular}

Figure 2. DLS of Sulfated $\mathrm{ZrO}_{2}$.

Normal reaction time without any catalyst for the synthesis of Benzimidazole was around 24 hour, but when we add little amount of $\mathrm{ZrO}_{2}$ powder reaction time was decreased to 90-100 minutes and yield was increased somewhat. While, activated Sulfated Zirconium dioxide powder were used in synthesis of benzimidazole, time of reaction was decrease to 4060 minutes and yield was high in compared to other similar catalysts which were referenced in introduction chapter.

After completion of reaction, rest of solution were treated with dimethyl chloride to dissolve all precipitated organic part, then centrifuged the solution to recover the Nano particles by decantation of solution. Particles were washed twice by cold dimethyl chloride before its further use.

Table 2. Reusability of $\mathrm{ZrO}_{2}$ and Sulfated $\mathrm{ZrO}_{2}$.

\begin{tabular}{|c|c|c|c|}
\hline Sr. No. & Nanocatalyst & $\begin{array}{c}\text { Average recovry } \\
\mathbf{Z r O} \mathbf{O}_{2}\end{array}$ & $\begin{array}{c}\text { Average recovry } \\
\text { Sulfated } \mathbf{Z r O}_{2}\end{array}$ \\
\hline 1 & $1^{\text {st }}$ Run & $80 \%$ & $85 \%$ \\
\hline 2 & $2^{\text {nd }}$ Run & $75 \%$ & $80 \%$ \\
\hline 3 & $3^{\text {rd }}$ Run & $70 \%$ & $76 \%$ \\
\hline 4 & $4^{\text {th }}$ Run & $65 \%$ & $72 \%$ \\
\hline
\end{tabular}




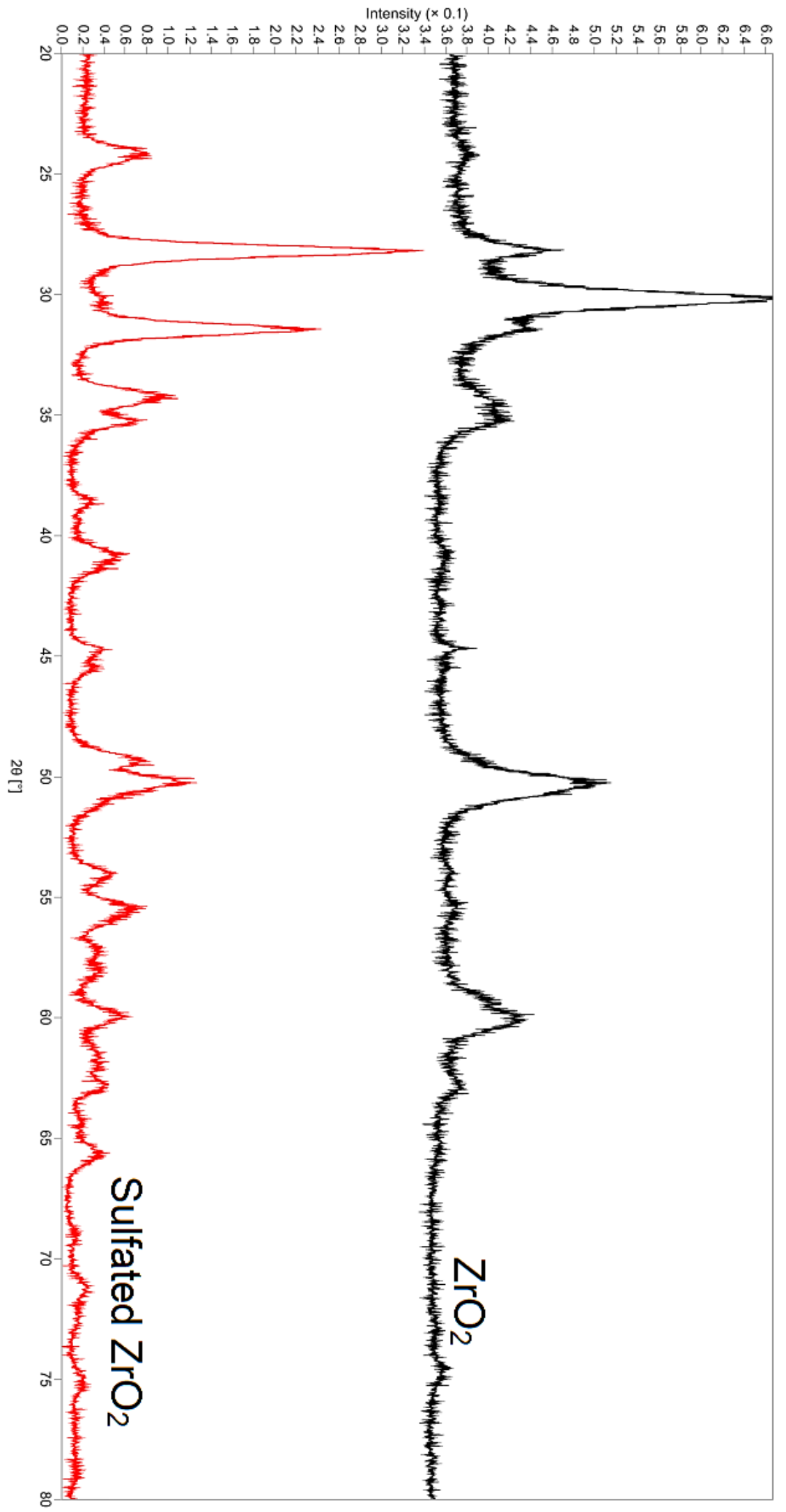

Figure 3. Powder $\mathrm{XRD}$ of $\mathrm{ZrO}_{2}$ and Sulfated $\mathrm{ZrO}_{2}$. 


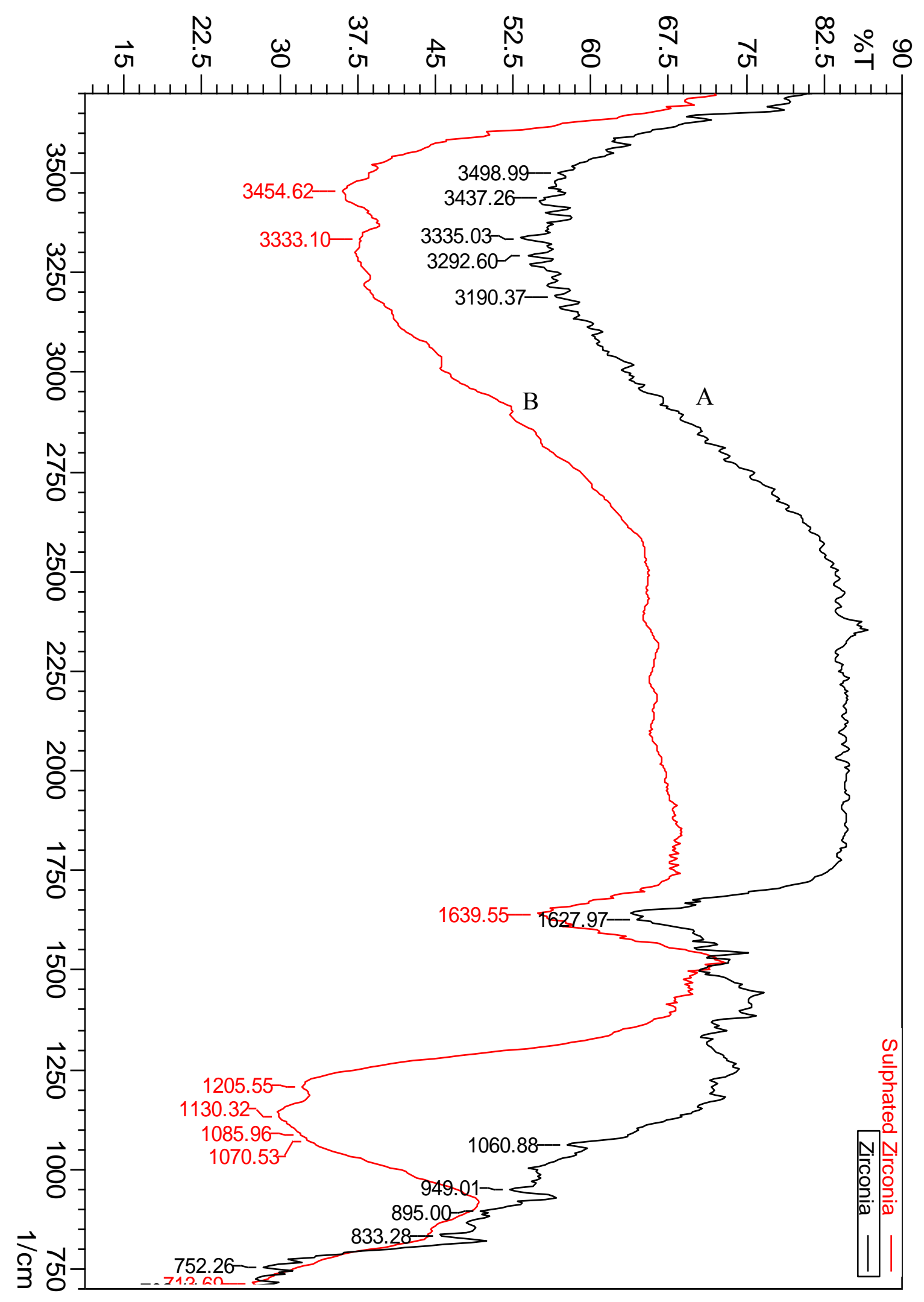

Figure 4. FT-IR of (A) $\mathrm{ZrO}_{2}$ (B) Sulphated $\mathrm{ZrO}_{2}$. 


\section{CONCLUSION}

We were able to synthesize $\mathrm{ZrO}_{2}$ and Sulfated $\mathrm{ZrO}_{2}$ (SZ) Nanoparticles by Sol-Gel method. The nanoparticles were characterized by IR, Powder XRD and DLS. The sizes of Nanoparticles were found in the range of $\sim 50-100 \mathrm{~nm}$. The prepared Nanoparticles shows higher catalytic activity compared to other methods described. Synthesis of benzodiazepines derivatives were characterized by Mass, FT-IR and NMR. However, using this SZ Nanoparticles shows greater reusability up till 3-4 times, hence beneficiary as catalyst.

\section{Acknowledgement}

Authors are thankful for UGC-BSR fellowship. Also acknowledge Physics department for pXRD data and NFDD, Rajkot for NMR data.

\section{References}

[1] D. J. Abraham, Burger's Medicinal Chemistry and Drug Discovery, John Wiley \& Sons, Inc., Hoboken, NJ, USA, 2003.

[2] R. G. Priest, U. V. Filho, R. Amrein, M. Skreta, Eds., Benzodiazepines, Springer Netherlands, Dordrecht, 1980.

[3] M. Abdollahi-Alibeik, I. Mohammadpoor-Baltork, Z. Zaghaghi, B. H. Yousefi, Catalysis Communications 9 (2008) 2496.

[4] J. A. L. Herbert, H. Suschitzky, Journal of the Chemical Society, Perkin Transactions 1 (1974) 2657.

[5] M. Curini, F. Epifano, M. C. Marcotullio, O. Rosati, Tetrahedron Letters 42 (2001) 3193.

[6] M. . Balakrishna, B. Kaboudin, Tetrahedron Letters 42 (2001) 1127.

[7] J. R. Sohn, H. W. Kim, Journal of Molecular Catalysis 52 (1989) 361.

[8] X. SONG, A. SAYARI, Catalysis Reviews 38 (1996) 329. 